\title{
Diferencial de salário entre trabalhadores nativos e migrantes na cafeicultura de Minas Gerais
}

\author{
Nilmar Diogo dos Reis" \\ Jaqueline Severino da Costa** \\ Arthur Alexandre Gonçalves *** \\ Luiz Gonzaga de Castro Junior ${ }^{* * * * *}$ \\ Renato Elias Fontes ${ }^{* * * * * * * *}$
}

\begin{abstract}
Resumo
O Brasil é o maior produtor e exportador mundial e o segundo consumidor de café no mundo. Por isso, o café é um dos produtos mais significativos para a economia brasileira. Em Minas Gerais, a produção é basicamente manual em virtude da topografia e, desta forma, utiliza muita mão de obra, que é composta por trabalhadores locais e imigrantes de outras regiões do Brasil, o que leva à discussão sobre se a renda auferida por esses trabalhadores é socialmente justa, sobretudo quando se comparam os rendimentos salariais da força de trabalho local com os rendimentos da força de trabalho em relação aos migrantes na cafeicultura. Os resultados encontrados apontaram para a existência de evidências de que os migrantes ganham um rendimento/hora superior aos trabalhadores nativos. Além disso, o efeito característica foi o maior responsável pelos ganhos superiores observados.
\end{abstract}

Palavras-chave: Discriminação de Oaxaca-Blinder. Oportunidades iguais. Migração.

" Doutorando em Administração, Universidade Federal de Lavras (UFLA). E-mail: nilmardiogo@ yahoo.com.br

*** Professora PhD em Economia, Universidade Federal de Lavras (UFLA). E-mail: jaqueline.s.costa@ ufla.br

**** Graduado em Administração, Universidade Federal de Lavras (UFLA). E-mail: artur.alexandre.goncalves@gmail.com

**** Professor PhD em Economia Aplicada, Universidade Federal de Lavras (UFLA). E-mail: lgcastro@ ufla.br

****** Professor PhD em Administração, Universidade Federal de Lavras (UFLA). E-mail: refontes@dga. ufla.br 


\section{Introdução}

O café possui posição de destaque na pauta de exportações brasileiras. O Brasil é o maior produtor e exportador mundial de café e ocupa o segundo lugar entre os maiores consumidores da bebida no mundo. Por isso, o café é um dos produtos mais significativos para a economia brasileira, com importante contribuição para 0 desenvolvimento nacional desde a indústria e serviço, além do próprio setor primário (SARAIVA et al., 2017). Destaca-se que a produção brasileira equivale a aproximadamente $30 \%$ da produção mundial e o país exporta por ano uma média de 34 milhões de sacas (CONAB, 2017).

As exportações de café verde têm propiciado divisas para o país e movimentaram um volume de recursos da ordem de US\$ 5,2 bilhões em 2017, contribuindo para a indução do produto interno bruto (PIB) brasileiro e impactando diretamente na empregabilidade de 8 milhões de pessoas (BRASIL, 2017).

Atualmente, a produção da espécie arábica está concentrada no estado de Minas Gerais, sendo este o maior estado produtor com um total de aproximadamente 24 milhões sacas em 2017, seguido por São Paulo, Espírito Santo e Bahia. Esses quatro estados concentram 85\% da produção nacional (CONAB, 2017).

Em Minas Gerais, a produção é basicamente manual em virtude da topografia e, por isso, utiliza muita mão de obra, que é composta por trabalhadores locais e imigrantes de outras regiões do Brasil, em especial da região nordeste do país (BINDA, 2013). Contudo, as condições de trabalho desses indivíduos carecem de análises, em especial neste momento, em que cresce a demanda por uma cafeicultura mais sustentável. Nesse sentido, surge a discussão sobre se a renda auferida por esses trabalhadores pode ser considerada socialmente justa, particularmente quando se comparam os rendimentos salariais da força de trabalho local com os da força de trabalho dos migrantes.

Este trabalho tem como objetivo aplicar a metodologia de decomposição de Oaxaca-Blinder (1973) ao longo do ciclo de vida do café. Para construção do Inventário Social do Ciclo de Vida, utilizou-se os dados secundários extraídos da Pesquisa Nacional por Amostra de Domicílios (PNAD), elaborada pelo Instituto Brasileiro de Geografia e Estatística (IBGE), do ano de 2015.

Além disso, mediante a importância do setor para o Brasil e, em particular, para o estado de Minas Gerais, este trabalho tem como objetivo analisar se existe diferencial de rendimento entre trabalhadores nativos e migrantes de outros 
estados do Brasil na produção de café em Minas Gerais. Ademais, a literatura, em sua maior parte, apresenta análises baseadas em técnicas que fazem uso de indicadores menos complexos; assim, neste trabalho, utilizou-se a decomposição de Oaxaca-Blinder para a mensurar essa diferenciação salarial na expectativa de obter análises mais contundentes.

\section{Referencial teórico}

Nesta seção, será apresentada a revisão de literatura, a qual consiste em duas partes: 1) apresentação do setor cafeeiro; 2) breve discussão a respeito da discriminação no mercado de trabalho brasileiro.

\section{O setor cafeeiro no Brasil}

No Brasil, as exportações de café ocupam a quinta posição entre as principais commodities. A área total cultivada no país com café (arábica e conilon) totaliza 2,13 milhões de hectares. Desse total, 319,17 mil hectares (15\%) estão em formação e 1,81 milhão de hectares (85\%) em produção. Desta última porcentagem, a área cultivada de arábica é de 1,73 milhão de hectares, correspondendo a $81 \%$ da área total existente, e a área de conilon é estimada em 398,8 mil hectares (CONAB, 2019).

O tipo de produção utilizado na propriedade rural produtora de café é caraterizado principalmente em função de seu relevo característico. A diversidade encontrada na topografia das principais regiões com aptidão para a cafeicultura no Brasil é decisiva para a determinação da tecnologia utilizada. Sendo assim, estão presentes nas realidades produtivas brasileiras os manejos manual, semimecanizado e mecanizado. A produção manual é caracterizada pela não utilização de máquinas automotoras, como tratores e colhedoras, nos processos de condução da lavoura e também na colheita, conforme o Boletim Ativos do Café (2015).

Ressalta-se que o uso de máquinas acopladas ao corpo humano, como roçadeiras, não caracteriza um processo produtivo ou uma atividade como mecanizada, da mesma forma que utilização de máquinas automotoras apenas no transporte interno de materiais e insumos. Os sistemas cuja produção é denominada semimecanizada são caracterizados pela utilização de máquinas automotoras nos processos de 
condução da lavoura. Apesar de serem realizadas atividades de forma mecanizada, como adubações e pulverizações, a colheita nesse tipo de produção é manual (BOLETIM ATIVOS DO CAFÉ, 2015).

Já nas propriedades onde a topografia e os recursos financeiros são favoráveis, é característico o tipo de produção mecanizado. Dessa forma, o Boletim Ativos do Café (2015) define esse tipo de manejo como os que se utilizam de máquinas automotoras nas atividades de condução da lavoura e nas atividades de colheita.

\section{Oportunidades iguais/discriminação}

A Organização Internacional do Trabalho (OIT), desde 1999, aspira por um conceito de trabalho decente, em que as oportunidades de acesso sejam iguais. Por meio de relações de trabalho que prezem pela equidade, sem discriminação de qualquer natureza, priorizando a dignidade da pessoa humana e a segurança OIT (1999).

Segundo a OIT (1999), a discriminação no trabalho resulta na intensificação do ciclo de pobreza, prejudicando a estabilidade política e a produtividade do trabalho. Dessa forma, a busca pela manutenção de relações de trabalho justas, nas quais a equidade se faça presente, é fundamental para o enfrentamento da pobreza, a manutenção da democracia, a igualdade social e, também, para o desenvolvimento sustentável.

Ainda de acordo com a OIT (2019), o gênero e a raça (ou cor) são fatores determinantes quando se trata das diferentes possibilidades de acesso a um bom emprego e suas respectivas condições trabalhistas, como remuneração justa e benefícios. Dessa forma, o gênero e a cor são fatores significativos quando se trata da forma como a pobreza será encarada pelos indivíduos e suas famílias, e também como vão conseguir ou não superar essa pobreza.

Diversos autores têm se dedicado aos estudos sobre as circunstâncias causadoras das diferenças salariais. Dentre eles, Giuberti e Menezes-Filho (2005) estudaram as diferenças de rendimento existentes entre o Brasil e os Estados Unidos nos anos de 1981, 1988 e 1996, naquilo que se refere à questão de gênero. De acordo com a conclusão dos autores, existem diferenças de rendimentos, mas elas não são explicadas pelas características masculinas e femininas.

No Brasil, os rendimentos salariais médios das mulheres correspondem a $68 \%$ do retorno masculino em 1981, enquanto que, em 1996, esta porcentagem passou 
para $80 \%$ do rendimento masculino. Nos Estados Unidos, o rendimento salarial das mulheres correspondia a $66 \%$ dos rendimentos auferidos pelos homens no ano de 1981, passando para 78\% em 1996. Tanto nos EUA como no Brasil, nos dois anos considerados, a diferença salarial sempre foi maior para pessoas do sexo masculino (GIUBERTI; MENEZES-FILHO, 2005). Na Áustria, cerca de 60\% da desigualdade salarial existente entre homens e mulheres não é explicada pelas diferentes características de produtividade. Em média, as mulheres austríacas ganham cerca de $11 \%$ a menos que os homens (BÖHEIM et al., 2013).

Grandner e Gstach (2015), ao analisar as diferenças salariais entre subgrupos da população da Alemanha e da Áustria, em 2008, encontraram diferenças salariais de cerca de $20 \%$ a $25 \%$ entre homens e mulheres, sendo que cerca de $15 \%$ dessas diferenças salariais se referem à discriminação, e o restante à diferença nas características.

No cenário brasileiro, Cirino e De Lima (2012) apontaram o valor da diferença de rendimentos do trabalho exercido na Região Metropolitana de Salvador (RMS) e na Região Metropolitana de Belo Horizonte (RMBH). Concluíram que, no ano de 2006, o retorno do trabalho é maior na RMBH em comparação com a RMS tanto para os homens quanto para as mulheres.

Mattei e Baço (2017) explicaram as diferenças salariais existentes no mercado de trabalho do estado de Santa Catarina nos anos 2000, 2007 e 2014. Os resultados apontaram para uma redução nas diferenças salariais entre homens e mulheres, o que indica que a discriminação contra as mulheres, entendida como a principal causa dessa diferenciação salarial, também diminuiu no período considerado.

Mais recentemente, Fonseca et al. (2018) analisaram a discriminação salarial com relação ao gênero e à cor para a Região Norte do Brasil em 2004 e 2013. O estudo concluiu que, apesar de o grau de discriminação por gênero e cor ter sido menor no período analisado, adesigualdade salarial permaneceu em razão do fator discriminatório.

Jacinto (2005), ao analisar os diferenciais de salários por gênero na indústria avícola da Região Sul do Brasil, buscou mensurar as diferenças de salários entre trabalhadores com graus de educação e ocupação semelhantes. O estudo concluiu que os homens apresentaram rendimentos salariais melhores em relação às mulheres.

Mattei e Baço (2017) analisaram a existência da diferença salarial entre os homens eas mulheres na indústria de transformação do estado de Santa Catarina. 
Os resultados mostraram que existe uma diferença salarial que favorece indivíduos do sexo masculino. Além disso, argumentam que a maior parte desta diferença é atribuída a fatores não explicados pelos atributos dos trabalhadores, o que pode indicar a existência de discriminação no setor de transformação.

Batista e Cacciamali (2009) estudaram o diferencial de salários entre homens e mulheres segundo a sua condição de migração. Eles constataram duas situações: na primeira, considera- se a Região Sudeste, onde a diferença de rendimentos por sexo é menor entre os indivíduos migrantes em comparação com os não migrantes; e na segunda situação, atenta-se para o resto do Brasil, onde a diferença de rendimentos por sexo é menor entre os indivíduos não migrantes em comparação com os migrantes.

Destaca-se, contudo, que a literatura a respeito da temática/problemática da desigualdade salarial entre migrantes e nativos no setor cafeeiro de modo geral e, especificamente, no estado de Minas Gerais ainda é incipiente. Isso reforça a necessidade da ampliação de estudos que abordem o problema.

\section{Procedimentos metodológicos}

Nesta seção, será apresentada a decomposição de Oaxaca-Blinder, que permite estimar a discriminação do mercado de trabalho, bem como a origem da fonte de dados usada nesta pesquisa.

\section{Oportunidades iguais/discriminação}

De forma a captar uma possível discriminação de migrantes e não migrantes nesse setor, foi realizada a decomposição de Oaxaca-Blinder. Este método consiste em estimar os rendimentos por hora dos trabalhadores na produção de café no estado de Minas Gerais. Por isso, torna-se necessário estimar a variável dependente (y) tanto para trabalhadores migrantes quanto para nativos do estado de Minas Gerais. A equação que capta as diferenças de rendimentos é do tipo:

$$
\ln \ln (y)=X^{\prime} \beta+v
$$


Em que $\ln \ln (y)$ é o vetor do logaritmo natural do rendimento do trabalhador; $X^{\prime} \beta$ é a matriz de variáveis explicativas; e $v$ é o vetor de erros aleatórios (CIRINO; DE LIMA, 2012).

Na matriz de variáveis explicativas X', foram consideradas as seguintes variáveis, que usualmente são utilizadas nas equações de rendimentos: escolaridade, idade, gênero, cor ou raça e posição na ocupação.

Essa metodologia pode ser aplicada a outros objetivos, por exemplo, ao se analisar as diferenças que ocorrem no comportamento de migrantes temporários e permanentes (ARISTEI, 2013). Para que seja possível a exemplificação da metodologia, será detalhada a decomposição da diferença salarial por gênero.

Segundo Oaxaca (1973) e Blinder (1973), para calcularmos a desigualdade salarial existente, por exemplo, entre homens e mulheres, o primeiro passo a ser estabelecido é a construção de equações mincerianas que, de acordo com Mincer (1974), é o modelo salarial de Mincer (1974), método utilizado para estimar retornos à educação, retornos à qualidade da educação, retornos à experiência, entre outros, como a equação de rendimento citada anteriormente.

Nas equações mincerianas, a variável dependente é o logaritmo do rendimento médio do trabalho principal e as variáveis independentes são idade, cor ou raça, posição na ocupação e escolaridade. Para trabalhadores locais, têm-se:

$$
\underline{Y_{L}}=\underline{X}_{L} \hat{\beta}_{L}
$$

E para migrantes:

$$
\underline{Y}_{M}=\underline{X}_{M} \hat{\beta}_{M}
$$

Em que $\underline{Y}$ é o rendimento médio de cada indivíduo pertencente a cada um dos grupos e os subscritos $L$ e $M$ representam homens e mulheres, respectivamente. A matriz $\underline{X}$ representa as características médias de cada grupo e o vetor $\beta$ simboliza o retorno dessas características.

Logo, calculamos os rendimentos contra-factuais das mulheres, caso elas sejam remuneradas iguais aos homens, ou seja, os retornos das características dos trabalhadores locais são transferidos para os trabalhadores migrantes:

$$
\tilde{Y}_{M}=X_{M} \hat{\beta}_{H}
$$


Em que $\tilde{Y}$ é o rendimento médio contra-factual dos trabalhadores migrantes. Desta forma, pode-se calcular a discriminação através da seguinte decomposição:

$$
\hat{Y}_{L}-\hat{Y}_{M}=\underline{X}_{H} \hat{\beta}_{H}-\underline{X}_{M} \hat{\beta}_{M}
$$

Dando sequência à decomposição, tem-se:

$$
\hat{Y}_{L}-\hat{Y}_{M}=\hat{\beta}_{M}\left(\underline{X}_{L}-\underline{X}_{M}\right)+\underline{X}_{M}\left(\hat{\beta}_{L}-\hat{\beta}_{M}\right)+\left(\underline{X}_{L}-\underline{X}_{M}\right)\left(\hat{\beta}_{L}-\hat{\beta}_{M}\right)
$$

Em que: $\widehat{Y}_{L}-\widehat{Y}_{M}$ representa a desigualdade total; $\hat{\beta}_{M}\left(\underline{X}_{L}-\underline{X}_{M}\right)$ consiste no efeito característica (parte da desigualdade devida às diferenças nas variáveis explicativas); $\underline{X}_{M}\left(\hat{\beta}_{L}-\hat{\beta}_{M}\right)$ se refere ao efeito preço (parte da desigualdade atribuída às diferenças nos $\beta$ 's), que é conhecida na literatura como termo de discriminação; e $\left(\underline{X}_{L}-\underline{X}_{M}\right)\left(\hat{\beta}_{L}-\hat{\beta}_{M}\right)$ se refere à interação entre ambos os termos da equação.

\section{Fonte de dados}

Os dados utilizados são referentes ao setor de café nas regiões brasileiras, sendo os dados caracterizados como secundários, extraídos da Pesquisa Nacional por Amostra de Domicílios (PNAD), elaborada pelo IBGE no ano de 2015.

\section{Resultados e discussão}

Para analisar o logaritmo, é necessário estimar uma equação de rendimentos, utiliza-se a forma logarítmica para incorporar a não linearidade na regressão. A não linearidade faz com que os retornos aumentem em uma proporção constante, o que é mais razoável do que em valor absoluto constante. Outro ponto é que essa forma de regressão faz com que se estreite a amplitude dos valores, tornando as estimativas menos sensíveis às observações extremas (outliers).

Assim, ao analisar o logaritmo do rendimento/hora dos trabalhadores na produção de café de Minas Gerais com o objetivo de fazer a decomposição de sua diferença e observar qual a representatividade de cada efeito no diferencial de rendimento total. Essa operação permite estimar qual a participação da discriminação entre trabalhadores nativos e migrantes na diferença total de rendimentos destes grupos. 
Na Tabela 1, é apresentada a decomposição da diferença do logaritmo do rendimento/hora entre os trabalhadores nativos e migrantes do setor de produção de café em Minas Gerais. Ao se utilizar da decomposição de Oaxaca-Blinder, expressa na Equação (6), foi possível estimar que a desigualdade total entre trabalhadores nativos e migrantes na produção de café em Minas Gerais foi de -0,2488. Esse coeficiente indica que os migrantes ganham em média $28 \%$ a mais se comparados aos trabalhadores nativos. Os resultados mostraram ainda que o rendimento/hora dos trabalhadores migrantes na produção de café em Minas Gerais é de $\mathrm{R} \$ 6,08$, enquanto que o rendimento/hora dos trabalhadores nativos foi de $\mathrm{R} \$ 4,75$ (Tabela 1).

Tabela 1 - Decomposição da diferença do logaritmo do rendimento/hora entre trabalhadores migrantes e trabalhadores nativos na produção de café em Minas Gerais

\begin{tabular}{l|c|c}
\hline \multicolumn{1}{c|}{$\begin{array}{c}\text { Diferencial do valor esperado do } \\
\text { logaritmo do rendimento/ hora }\end{array}$} & Coeficiente & Rendimento hora \\
\hline Trabalhador migrante & $1,806475(0,0382)^{\star}$ & 6,08 \\
Trabalhador Nativo & $1,5575(0,0974)^{\star}$ & 4,75 \\
Diferença & $-0,2488(0,1047)^{\star \star}$ & 1,28 \\
Efeito características & $0,3045(0,2097)^{\star}$ & $\%$ Diferença \\
Efeito preço & $-0,2181(0,1063)^{\star \star}$ & $-122,38$ \\
Interação & $-0,3352(0,1116)^{\star}$ & 87,66 \\
\hline
\end{tabular}

Fonte: elaboração dos autores com base nos dados da PNAD 2015.

Notas: *Significativo a 1\%; **Significativo a 5\%; variáveis de controle-educação, idade, idade ao quadrado (proxy da experiência), gênero, cor ou raça, com carteira assinada e sem carteira assinada.

Enquanto o efeito característica é parte da desigualdade atribuída às diferenças nas variáveis explicativas, o efeito preço mensura o retorno de cada característica produtiva. Ao se medir o efeito simultâneo das diferenças nas características e nas diferenças nos retornos salariais, tem-se o efeito de interação (JANN, 2008).

O efeito característica representa $-122,38 \%$ do diferencial de rendimento entre migrantes e nativos - que é o percentual de diferença calculado através do respectivo coeficiente dividido pelo coeficiente da diferença -, ou seja, a diferença nos atributos dos trabalhadores fez com que os migrantes recebessem cerca de 35,6\% - da qual esta porcentagem é calculada através da subtração do exponencial do coeficiente menos um -, a mais que os trabalhadores nativos na produção de café em Minas Gerais. 
O efeito característica pode ser o maior responsável pela diferenciação salarial entre trabalhadores nativos e trabalhadores migrantes. Possivelmente, os trabalhadores migrantes podem ser mais produtivos do que os trabalhadores nativos e apresentar nível de escolaridade ou qualificação técnica superiores em relação a eles.

$\mathrm{O}$ efeito preço se refere à diferença nos retornos das características produtivas e é o grande responsável pela diferenciação salarial entre trabalhadores nativos e trabalhadores migrantes. O efeito preço teve como impacto o aumento do diferencial de rendimentos. Ele representa $87,66 \%$ do diferencial total de rendimentos entre trabalhadores nativos e trabalhadores migrantes na produção de café em Minas Gerais.

Os resultados do efeito preço apresentaram um retorno médio do trabalho dos nativos superior ao do trabalho dos migrantes. A parcela do diferencial de rendimentos entre os trabalhadores nativos e os trabalhadores migrantes, que é atribuído às diferenças de retorno das características produtivas, faz com que os trabalhadores migrantes recebam $19,6 \%$ a menos que os trabalhadores nativos. Isso implica em dizer que não existe discriminação de rendimento/hora em favor dos trabalhadores migrantes. Conclui-se, a partir daí, que os trabalhadores migrantes possuem maior destreza, produtividade ou escolaridade em relação aos trabalhadores nativos que trabalham no setor cafeeiro.

O termo de interação mostrou-se significativo na análise, pois representou uma possibilidade de aumento no diferencial total de $134,72 \%$. Isso indica que o retorno médio dos trabalhadores migrantes é $28,5 \%$ menor se comparado aos trabalhadores nativos.

\section{Conclusões}

Este estudo teve como objetivo analisar a existência de uma possível discriminação salarial entre trabalhadores migrantes e trabalhadores nativos na produção de café em Minas Gerais através da decomposição de Oaxaca-Blinder. Para realizar este estudo, foi necessário estimar os rendimentos por hora trabalhada no setor produtivo de café em Minas Gerais. A base de dados utilizada foi a PNAD 2015.

Os resultados encontrados apontaram para a existência de evidências de que os migrantes ganham um rendimento/hora superior aos trabalhadores nativos. Além disso, o efeito característica teve como característica o fator de maior relevância pelos ganhos superiores observados nos trabalhadores migrantes. 
Na produção de café em Minas Gerais, há evidências da predileção pela mão de obra de trabalhadores migrantes, possivelmente em razão do fato de o trabalhador se deslocar e aproveitar o período da colheita para ter uma renda maior e enviá-la a sua família na região de origem.

Entretanto, existem algumas limitações que precisam ser evidenciadas neste trabalho. A primeira delas se refere ao problema de seleção amostral, pois observou-se somente o rendimento das pessoas que estão trabalhando de forma remunerada. Uma possível solução seria utilizar o modelo de seleção amostral desenvolvido por Heckmman, o Tobit II.

Uma segunda limitação se refere ao problema de variáveis omitidas e que podem ter impactos não inicialmente previstos na amostra. A escolha, a produtividade, a motivação e a habilidade são exemplos de variáveis que não foram controladas na regressão, mas que podem determinar o rendimento das pessoas, além de estarem possivelmente relacionadas às variáveis explicativas utilizadas no modelo.

\section{Agradecimentos}

Os autores agradecem, de forma irrestrita, aos revisores do VII Simpósio da Ciência do Agronegócio - 2020, à Coordenação de Aperfeiçoamento de Pessoal de Nível Superior (Capes), ao Conselho Nacional de Desenvolvimento Científico e Tecnológico (CNPq), à Fundação de Amparo à Pesquisa do Estado de Minas Gerais (FAPEMIG) e ao Centro de Inteligência em Mercados (CIM-UFLA). 


\title{
Diferencial salarial entre trabajadores nativos y migrantes en la producción de café en Minas Gerais
}

\begin{abstract}
Resumen
Brasil es el mayor productor y exportador del mundo y el segundo mayor consumidor de café del mundo. Por eso, el café es uno de los productos más importantes para la economía brasileña. En Minas Gerais, la producción es básicamente manual debido a la topografía y, por lo tanto, utiliza mucha mano de obra que está conformada por trabajadores locales e inmigrantes de otras regiones de Brasil, lo que lleva a la discusión de si el ingreso de estos trabajadores es socialmente justo, especialmente cuando se compara el ingreso salarial de la fuerza laboral local con la fuerza laboral en relación con los migrantes en el cultivo del café. Los resultados encontrados apuntan a la existencia de evidencia de que los migrantes obtienen un ingreso por horas más alto que los trabajadores nativos. El efecto característico fue en gran parte responsable de las mayores ganancias observadas.
\end{abstract}

Palabras clave: Discriminación Oaxaca-Blinder. Oportunidades iguales. Migración.

\section{Wage differential between native and migrant workers in the cafeiculture of Minas Gerais}

\begin{abstract}
Brazil is the world's largest producer and exporter and the second largest consumer of coffee in the world. For this reason, coffee is one of the most significant products for the Brazilian economy. In Minas Gerais, production is basically manual due to the topography and, therefore, uses a lot of labor that is made up of local workers and immigrants from other regions of Brazil, which leads to the discussion of whether the income earned by these workers is socially just, especially when comparing the wage income of the local labor force to the labor force in relation to migrants in coffee farming. The results found pointed to the existence of evidence that migrants earn a higher hourly income than native workers. In addition, the characteristic effect was largely responsible for the largest gains seen.
\end{abstract}

Keywords: Oaxaca-Blinder discrimination. Equal opportunities. Migration. 


\section{Referências}

ARISTEI, D. A Blinder-Oaxaca decomposition for double-hurdle models with an application to migrants' remittance behaviour. Applied Economics Letters, v. 20, n. 18, p. 1665-1672, 2013.

BATISTA, N. N. F.; CACCIAMALI, M. C. Diferencial de salários entre homens e mulheres segundo a condição de migração. Revista Brasileira de Estudos de População, v. 26, n. 1, p. 97-115, 2009.

BINDA, R. J. Doce exploração: percepções do trabalhador rural do setor sucroenergético da microrregião nordeste do Espírito Santo sobre dignidade em sua atividade laboral. Dissertação (Mestrado) - Faculdade de Direito de Vitória, Vitória, 2013.

BLINDER, A. S. Wage discrimination: reduced form and structural variables. Journal of Human Resources, v. 8, p. 436-455, 1973.

BOLETIM ATIVOS DO CAFÉ. Superintendência Técnica da CNA e Centro de Inteligência em Mercados (CIM) da Universidade Federal de Lavras (UFLA), ano 9, Edição 20, 2015. Disponível em: https://www.cnabrasil.org.br/assets/arquivos/boletins/ativos-cafe- 20_0.83833200\%20 1514916999.pdf. Acesso em: 05 jun. 2020.

BÖHEIM, R. et al. The distribution of the gender wage gap in Austria: evidence from matched employer-employee data and tax records. Journal for Labour Market Research, v. 46, n. 1, p. 19$34,2013$.

BRASIL. Ministério da Agricultura, Pecuária e Abastecimento (MAPA). Café no Brasil. 2017. Disponível em: http://antigo.agricultura.gov.br/assuntos/politica- agricola/cafe/cafeicultura-brasileira. Acesso em: 05 jun. 2020.

COMPANHIA NACIONAL DE ABASTECIMENTO (CONAB). Acompanhamento da safra brasileira de café. v. 5, Safra 2019, n. 3, Terceiro levantamento, Brasília, p. 1-48, set. 2019.

COMPANHIA NACIONAL DE ABASTECIMENTO (CONAB). Acompanhamento safra brasileira de café, v. 4, Safra 2017, n. 4, Quarto levantamento, Brasília, p. 1-84, dez. 2017.

CIRINO, J. F.; de LIMA, J. E. Diferenças de rendimento entre as regiões metropolitanas de Belo Horizonte e Salvador: uma discussão a partir da decomposição de Oaxaca-Blinder. Revista Econômica do Nordeste, v. 43, n. 2, p. 371-390, 2012.

FONSECA, M. R. et al. Diferenças salariais e discriminação por gênero e cor na região norte do Brasil. Revista de Políticas Públicas, v. 21, n. 2, p. 739-760, 2018.

GIUBERTI, A. C.; MENEZES-FILHO, N. Discriminação de rendimentos por gênero: uma comparação entre o Brasil e os Estados Unidos. Economia Aplicada, v. 9, n. 3, p. 369-384, 2005.

GRANDNER, T.; GSTACH, D. Decomposing wage discrimination in Germany and Austria with counterfactual densities. Empirica, v. 42, n. 1, p. 49-76, 2015.

JACINTO, P. D. A. Diferenciais de salários por gênero na indústria avícola da região Sul do Brasil: uma análise com microdados. Revista de Economia e Sociologia Rural, v. 43, n. 3, p. 529-555, 2005.

JANN, B. The Blinder-Oaxaca decomposition for linear regression models. The Stata Journal, v. 8, n. 4, p. 453-479, 2008.

Teoria e Evidência Econômica - a. 26, n. 55, p. 172-185, jul./dez. 2020 
MATTEI, T. F.; BAÇO, F. M. B. Análise das desigualdades salariais entre homens e mulheres no mercado de trabalho de Santa Catarina. DRd-Desenvolvimento Regional em Debate, v. 7, n. 2, p. 96-117, 2017.

MINCER, J. Schooling, Experience, and Earnings. Human Behavior \& Social Institutions, n. 2, 1974.

OAXACA, R. L. Male-female wage differentials in urban labor markets. International Economic Review, v. 14, p. 693-709, 1973.

ORGANIZAÇÃO INTERNACIONAL DO TRABALHO (OIT). 1999. Disponível em: https://www. ilo.org/public/english/standards/declaration/ declaration_portuguese.pdf. Acesso em: 01 mar. 2020.

ORGANIZAÇÃO INTERNACIONAL DO TRABALHO (OIT). 2019. Disponível em: https://www. ilo.org/brasilia/temas/g\%C3\%AAnero-e- ra\%C3\%A7a/lang--pt/index.htm. Acesso em: 05 mar. 2020.

SARAIVA, C. E. D. A. B.; FERNANDES, A. M.; LIMA, A. P. A.; da COSTA, L. T.; CUNHA, C. N. Competitividade na Cafeicultura Brasileira. In: MOSTRA DE INICIAÇÃO CIENTÍFICA, PÓS-GRADUAÇÃO, PESQUISA E EXTENSÃO, 17. 2017. 